\title{
MAGNETIC ORDERING OF ANTIFERROMAGNETS ON A SPATIALLY ANISOTROPIC TRIANGULAR LATTICE
}

\author{
R. F. Bishop and P. H. Y. Li \\ School of Physics and Astronomy, Schuster Building, The University of Manchester, \\ Manchester, M13 9PL, UK \\ D. J. J. Farnell \\ Health Methodology Research Group, School of Community-Based Medicine, \\ Jean McFarlane Building, University Place, The University of Manchester, M13 9PL, UK \\ C. E. Campbell \\ School of Physics and Astronomy, University of Minnesota, 116 Church Street SE, \\ Minneapolis, MN 55455, USA \\ Received Day Month Year \\ Revised Day Month Year
}

\begin{abstract}
We study the spin-1/2 and spin- $1 J_{1}-J_{2}^{\prime}$ Heisenberg antiferromagnets (HAFs) on an infinite, anisotropic, two-dimensional triangular lattice, using the coupled cluster method. With respect to an underlying square-lattice geometry the model contains antiferromagnetic $\left(J_{1}>0\right)$ bonds between nearest neighbours and competing $\left(J_{2}^{\prime}>0\right)$ bonds between next-nearest-neighbours across only one of the diagonals of each square plaquette, the same diagonal in each square. In a topologically equivalent triangular-lattice geometry the model has two sorts of nearest-neighbour bonds, with $J_{2}^{\prime} \equiv \kappa J_{1}$ bonds along parallel chains and with $J_{1}$ bonds providing an interchain coupling. The model thus interpolates between an isotropic HAF on the square lattice at one extreme $(\kappa=0)$ and a set of decoupled chains at the other $(\kappa \rightarrow \infty)$, with the isotropic HAF on the triangular lattice in between at $\kappa=1$. For the spin-1/2 $J_{1}-J_{2}^{\prime}$ model, we find a weakly first-order (or possibly second-order) quantum phase transition from a Néel-ordered state to a helical state at a first critical point at $\kappa_{c_{1}}=0.80 \pm 0.01$, and a second critical point at $\kappa_{c_{2}}=1.8 \pm 0.4$ where a first-order transition occurs between the helical state and a collinear stripeordered state. For the corresponding spin-1 model we find an analogous transition of the second-order type at $\kappa_{c_{1}}=0.62 \pm 0.01$ between states with Néel and helical ordering, but we find no evidence of a further transition in this case to a stripe-ordered phase.
\end{abstract}

Keywords: Quantum magnet; quantum phase transition; coupled cluster method.

\section{Introduction}

Arrays of strongly interacting quantum spins arranged on a regular periodic lattice are among the conceptually simplest to describe via a Hamiltonian, but are amongst the most challenging many-body systems in terms of a theoretical derivation of their ground- and excited-state properties from first principles. They have 
become the subject of intense interest in recent years, especially since many such models give a good description of real magnetic materials that can be studied experimentally. Particular attention has focussed on two-dimensional (2D), spin-1/2 Heisenberg antiferromagnets (HAFs), for which the effects of quantum fluctuations are usually larger than for their counterparts with higher spatial dimensionality or with a greater value of the spin quantum number, $s>1 / 2$. Of special interest has been the interplay between frustration (either geometric or dynamic in origin) and quantum fluctuations in determining the ground-state (gs) phase diagram for such models. While simple models of this kind are well understood in the absense of frustration, ${ }^{1}$ their frustrated counterparts present a much greater theoretical challenge. This is particularly so when there are two or more terms in the interaction Hamiltonian that compete with one another to produce different types of ordering. In particular the zero-temperature $(T=0)$ phase transitions between magnetically ordered quasiclassical phases and novel (magnetically disordered) quantum paramagnetic phases ${ }^{2,3}$ have attracted huge interest in recent years, both as prototypical examples of quantum phase transitions of various types and because of the possible fabrication of real magnets that exhibit such phases, and which may have novel properties.

A particularly well studied such model is the frustrated spin-1/2 $J_{1}-J_{2}$ model on the square lattice with nearest-neighbour $(\mathrm{NN})$ bonds $\left(J_{1}\right)$ and next-nearestneighbour (NNN) bonds $\left(J_{2}\right)$, for which it is now well accepted that there exist two phases exhibiting magnetic long-range order (LRO) at small and at large values of $\alpha \equiv J_{2} / J_{1}$ respectively, separated by an intermediate quantum paramagnetic phase without magnetic LRO in the parameter regime $\alpha_{c_{1}}<\alpha<\alpha_{c_{2}}$, where $\alpha_{c_{1}} \approx 0.4$ and $\alpha_{c_{2}} \approx 0.6$. For $\alpha<\alpha_{c_{1}}$ the gs phase exhibits Néel magnetic LRO, whereas for $\alpha>\alpha_{c_{2}}$ it exhibits collinear stripe LRO. We have recently studied this $2 \mathrm{D}$ spin- $1 / 2$ model exhaustively by extending it to include anisotropic interactions in either real (crystal lattice) space ${ }^{4}$ or in spin space. ${ }^{5}$ We showed in particular how the coupled cluster method (CCM) provided for this highly frustrated model what is perhaps now the most accurate microscopic description. The interested reader is referred to Refs. $[4,5]$ and references cited therein for further details of the model and the method.

\section{The Model}

Since the CCM has proven so successful in the above applications we now apply it to the seemingly similar $2 \mathrm{D} J_{1}-J_{2}^{\prime}$ model that has been studied recently by other means for the spin- $1 / 2$ case. ${ }^{6-11}$ Its Hamiltonian is written as

$$
H=J_{1} \sum_{\langle i, j\rangle} \mathbf{s}_{i} \cdot \mathbf{s}_{j}+J_{2}^{\prime} \sum_{[i, k]} \mathbf{s}_{i} \cdot \mathbf{s}_{k}
$$

where the operators $\mathbf{s}_{i} \equiv\left(s_{i}^{x}, s_{i}^{y}, s_{i}^{z}\right)$ are the spin operators on lattice site $i$ with $\mathbf{s}_{i}^{2}=s(s+1)$, and where we study both the cases $s=1 / 2$ and $s=1$ here. On 

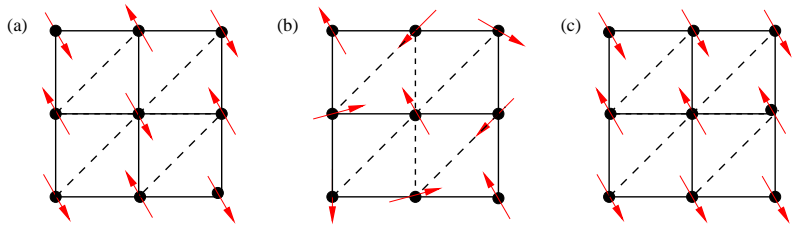

Fig. 1. $J_{1}-J_{2}^{\prime}$ model; $-J_{1} ;---J_{2}^{\prime}$; (a) Néel state, (b) spiral state, (c) stripe state.

the square lattice the sum over $\langle i, j\rangle$ runs over all distinct NN bonds, but the sum over $[i, k]$ runs only over one half of the distinct NNN bonds with equivalent bonds chosen in each square plaquette, as shown explicitly in Fig. 1. (By contrast, the $J_{1}-J_{2}$ model discussed above includes all of the diagonal NNN bonds.) We shall be interested here only in the case of competing (or frustrating) antiferromagnetic bonds $J_{1}>0$ and $J_{2}^{\prime}>0$, and henceforth for all of the results shown we set $J_{1} \equiv 1$. Clearly, the model may be described equivalently as a Heisenberg model on an anisotropic triangular lattice in which each triangular plaquette contains two NN $J_{1}$ bonds and one NN $J_{2}^{\prime}$ bond. The model thus interpolates continuously between HAFs on a square lattice $\left(J_{2}^{\prime}=0\right)$ and on a triangular lattice $\left(J_{2}^{\prime}=J_{1}\right)$. Similarly, when $J_{1}=0$ (or $J_{2}^{\prime} \rightarrow \infty$ in our normalization with $J_{1} \equiv 1$ ) the model reduces to uncoupled 1D chains (along the chosen diagonals on the square lattice). The case $J_{2}^{\prime} \gg 1$ thus corresponds to weakly coupled 1D chains, and hence the model also interpolates between 1D and 2D.

In the classical case (correponding to the asympototic limit where the spin quantum number $s \rightarrow \infty)$ the $J_{1}-J_{2}^{\prime}$ model has only two classical gs phases. For $J_{2}^{\prime}<\frac{1}{2} J_{1}$ the gs phase is Néel ordered, as shown in Fig. 1(a), whereas for $J_{2}^{\prime}>\frac{1}{2} J_{1}$ it has spiral order, as shown in Fig. 1(b), wherein the spin direction at lattice site $(i, j)$ points at an angle $\alpha_{i j}=\alpha_{0}+(i+j) \alpha_{\mathrm{cl}}$, with $\alpha_{\mathrm{cl}}=\cos ^{-1}\left(-\frac{J_{1}}{2 J_{2}^{\prime}}\right) \equiv \pi-\phi_{\mathrm{cl}}$. The pitch angle $\phi_{\mathrm{cl}}=\cos ^{-1}\left(\frac{J_{1}}{2 J_{2}^{\prime}}\right)$ thus measures the deviaton from Néel order, and it varies from zero for $2 J_{2}^{\prime} / J_{1} \leq 1$ to $\frac{1}{2} \pi$ as $J_{2}^{\prime} / J_{1} \rightarrow \infty$, as shown in Fig. 3 below. When $J_{2}^{\prime}=J_{1}$ we regain the classical 3 -sublattice ordering on the triangular lattice with $\alpha_{\mathrm{cl}}=\frac{2}{3} \pi$. The classical phase transition at $J_{2}^{\prime}=\frac{1}{2} J_{1}$ is of continuous (second-order) type, with the gs energy and its derivative both continuous.

In the limit of large $J_{2}^{\prime} / J_{1}$ the above classical limit represents a set of decoupled 1D HAF chains (along the diagonals of the square lattice) with a relative spin orientation between neighbouring chains that approaches $90^{\circ}$. In fact, of course, there is complete degeneracy at the classical level in this limit between all states for which the relative ordering directions of spins on different HAF chains are arbitrary. Clearly the exact spin-1/2 limit should also be a set of decoupled HAF chains, as given by the exact Bethe ansatz solution. ${ }^{12}$ However, one might expect that this degeneracy could be lifted by quantum fluctuations, via the well-known phenomenon of order by disorder. ${ }^{13}$ Just such a phase is known to exist in the $J_{1}-J_{2}$ model $^{4,5}$ for values of $J_{2} / J_{1} \gtrsim 0.6$, where it is the so-called collinear stripe phase in which, on 
the square lattice, spins along (say) the rows in Fig. 1 order ferromagnetically while spins along the columns and diagonals order antiferromagnetically, as shown in Fig. $1(\mathrm{c})$. We note that a recent renormalization-group (RG) ${ }^{10}$ analysis of the spin- $1 / 2$ $J_{1}-J_{2}^{\prime}$ model considered here has predicted that just such a collinear stripe phase also exists in this case for values of $J_{2}^{\prime} / J_{1}$ greater than some critical value which was not calculated in that analysis. Our own results are presented below.

\section{The Coupled Cluster Method Formalism}

The CCM (see, e.g., Refs. [14-16] and references cited therein) that we employ here is one of the most powerful and most versatile modern techniques in quantum manybody theory. It has been applied very successfully to various quantum magnets (see Refs. $[4,5,16-22]$ and references cited therein), as we now briefly outline below. The starting point for any CCM calculation is to select a normalized model or reference state $|\Phi\rangle$. It is often convenient to take a classical ground state (GS) as a model state for CCM calculations of quantum spin systems. Accordingly our model states here include the Néel and spiral states, although we also employ the nonclassical stripe state. It is very convenient to treat each site on an equal footing, and in order to do so we perform a mathematical rotation of the local axes on each lattice site such that all spins in every reference state align along the negative $z$-axis. The Schrödinger ground-state ket and bra equations are $H|\Psi\rangle=E|\Psi\rangle$ and $\langle\tilde{\Psi}| H=E\langle\tilde{\Psi}|$ respectively. The CCM parametrizes these exact quantum gs wave functions in the forms $|\Psi\rangle=\mathrm{e}^{S}|\Phi\rangle$ and $\langle\tilde{\Psi}|=\langle\tilde{\Phi}| \tilde{S} \mathrm{e}^{-S}$. The correlation operators $S$ and $\tilde{S}$ are expressed as $S=\sum_{I \neq 0} \mathcal{S}_{I} C_{I}^{+}$and $\tilde{S}=1+\sum_{I \neq 0} \tilde{\mathcal{S}}_{I} C_{I}^{-}$, where $C_{0}^{+} \equiv 1$, the unit operator, and $C_{I}^{+} \equiv\left(C_{I}^{-}\right)^{\dagger}$ is one of a complete set of multispin creation operators with respect to the model state (with $C_{I}^{-}|\Phi\rangle=0=\langle\Phi| C_{I}^{+} ; \forall I \neq 0$ ), generically written as $C_{I}^{+} \equiv s_{i_{1}}^{+} s_{i_{2}}^{+} \cdots s_{i_{n}}^{+}$, in terms of the spin-raising operators $s_{i}^{+} \equiv s_{i}^{x}+s_{i}^{y}$ on lattice sites $i$. We note that $\langle\tilde{\Psi} \mid \Psi\rangle=\langle\Phi \mid \Psi\rangle=\langle\Phi \mid \Phi\rangle \equiv 1$.

The ket- and bra-state correlation coefficients $\left(\mathcal{S}_{I}, \tilde{\mathcal{S}}_{I}\right)$ are calculated by requiring the expectation value $\bar{H} \equiv\langle\tilde{\Psi}|H| \Psi\rangle$ to be a minimum with respect to each of them. This immediately yields the coupled set of equations $\left\langle\Phi\left|C_{I}^{-} \mathrm{e}^{-S} H \mathrm{e}^{S}\right| \Phi\right\rangle=0$ and $\left\langle\Phi\left|\tilde{S}\left(\mathrm{e}^{-S} H \mathrm{e}^{S}-E\right) C_{I}^{+}\right| \Phi\right\rangle=0 ; \forall I \neq 0$, which we solve for the correlation coefficients $\left(\mathcal{S}_{I}, \tilde{\mathcal{S}}_{I}\right)$. We may then calculate the gs energy from the relation $E=\left\langle\Phi\left|\mathrm{e}^{-S} H \mathrm{e}^{S}\right| \Phi\right\rangle$, and the gs staggered magnetization $M$ from the relation $M \equiv-\frac{1}{N}\left\langle\tilde{\Psi}\left|\sum_{i=1}^{N} s_{i}^{z}\right| \Psi\right\rangle$ which holds in the local (rotated) spin coordinates on each lattice site $i$. We note that we work from the outset in the $N \rightarrow \infty$ limit, where $N$ is the number of spins on the lattice.

The CCM formalism is exact if a complete set of multispin configurations $\{I\}$ with respect to the model state is included in the calculation. However, it is necessary in practice to use approximation schemes to truncate the expansions in configurations $\{I\}$ of the correlation operators $S$ and $\tilde{S}$. For the case of $s=1 / 2$ we employ here, as in our previous work, ${ }^{4,5,16-22}$ the localized LSUB $m$ scheme in which all possible multi-spin-flip correlations over different locales on the lattice defined by 


\begin{tabular}{|c|c|c|c|c|c|}
\hline \multicolumn{6}{|c|}{$\begin{array}{l}\text { Table } 1 \text {. Numbers of fundamental configurations ( } \sharp \text { f.c.) } \\
\text { for } s=1 / 2 \text { and } s=1 \text { in various CCM approximations. }\end{array}$} \\
\hline \multicolumn{3}{|c|}{$s=1 / 2$} & \multicolumn{3}{|c|}{$s=1$} \\
\hline \multirow[t]{2}{*}{ Scheme } & \multicolumn{2}{|c|}{$\sharp$ f.c. } & \multirow[t]{2}{*}{ Scheme } & \multicolumn{2}{|c|}{$\sharp$ f.c. } \\
\hline & stripe & spiral & & stripe & spiral \\
\hline LSUB2 & 2 & 3 & SUB2-2 & 2 & 4 \\
\hline LSUB3 & 4 & 14 & SUB3-3 & 4 & 26 \\
\hline LSUB4 & 27 & 67 & SUB4-4 & 60 & 189 \\
\hline LSUB5 & 95 & 370 & SUB5-5 & 175 & 1578 \\
\hline LSUB6 & 519 & 2133 & SUB6-6 & 2996 & 14084 \\
\hline LSUB7 & 2617 & 12878 & SUB7-7 & 11778 & 131473 \\
\hline LSUB8 & 15337 & 79408 & - & - & - \\
\hline
\end{tabular}

$m$ or fewer contiguous lattice sites are retained. The numbers of such fundamental configurations (viz., those that are distinct under the symmetries of the Hamiltonian and of the model state $|\Phi\rangle$ ) that are retained for the stripe and spiral states of the current model in various LSUB $m$ approximations on the triangular lattice are shown in Table 1.

We note next that the number of fundamental LSUB $m$ configurations for $s=1$ becomes appreciably higher than for $s=1 / 2$, since each spin on each site $i$ can now be flipped twice by the spin-raising operator $s_{i}^{+}$. Thus, for the $s=1$ model it is more practical to use the alternative $\mathrm{SUB} n-m$ scheme, where $m$ is the size of the locale on the lattice and $n$ is the maximum number of spin-flips. Hence all correlations involving up to $n$ spin flips spanning a range of no more than $m$ adjacent lattice sites are retained. ${ }^{16}$ In our case we set $m=n$, and hence employ the SUB $m-m$ scheme as in our previous work (see, e.g., Refs. [16,22] and references cited therein). More generally, the LSUB $m$ scheme is thus equivalent to the SUB $n-$ $m$ scheme for $n=2 s m$. Hence, $\mathrm{LSUB} m \equiv \mathrm{SUB} 2 s m-m$. For $\mathrm{s}=1 / 2, \mathrm{LSUB} m \equiv$ $\mathrm{SUB} m-m$; whereas for $s=1, \mathrm{LSUB} m \equiv \mathrm{SUB} 2 m-m$. The numbers of fundamental configurations retained at various $\mathrm{SUB} m-m$ levels for the $s=1$ model are also shown in Table 1.

In order to solve the corresponding coupled sets of CCM bra- and ket-state equations we use parallel computing. ${ }^{23}$ The highest CCM level that we can reach here, even with massive parallelization and the use of supercomputing resources, is LSUB8 for $s=1 / 2$ and SUB7-7 for $s=1$. For example, to obtain a single data point (i.e., for a given value of $J_{2}^{\prime}$, with $J_{1}=1$ ) for the spiral phase at the LSUB8 level for the $s=1 / 2$ case typically required about $0.3 \mathrm{~h}$ computing time using 600 processors simultaneously. It similarly required about $0.25 \mathrm{~h}$ for the spiral phase at the SUB7-7 level for the $s=1$ case.

It is important to note that we never need to perform any finite-size scaling, since all CCM approximations are automatically performed from the outset in the infinite-lattice limit, $N \rightarrow \infty$, where $N$ is the number of lattice sites. However, we do 
need as a last step to extrapolate to the $m \rightarrow \infty$ limit in the LSUB $m$ or SUB $m-m$ truncation index $m$. We use here the well-tested ${ }^{17,18}$ empirical scaling laws

$$
\begin{gathered}
E / N=a_{0}+a_{1} m^{-2}+a_{2} m^{-4}, \\
M=b_{0}+b_{1} m^{-1}+b_{2} m^{-2} .
\end{gathered}
$$

\section{Results and Discussion}

We report here on CCM calculations for the spin- $1 / 2$ and spin- $1 J_{1}-J_{2}^{\prime}$ model Hamiltonians of Eq. (1) for given parameters $\left(J_{1}=1, J_{2}^{\prime}\right)$, based respectively on the Néel, spiral and stripe states as CCM model states. We note that, as has been well documented in the past, ${ }^{24}$ the LSUBm (and SUB $m-m$ ) data for both the gs energy per spin $E / N$ and the on-site magnetization $M$ converge differently for the even- $m$ and the odd- $m$ sequences, similar to what is frequently observed in perturbation theory. ${ }^{25}$ Since, as a general rule, it is desirable to have at least $(n+1)$ data points to fit to any fitting formula that contains $n$ unknown parameters, we prefer to have at least 4 results to fit to Eqs. (2) and (3). Hence, for most of our extrapolated results below we use the even LSUB $m$ sequence with $m=\{2,4,6,8\}$ for the spin- $1 / 2$ case. For the spin- 1 case we use the SUB $m-m$ sequences with $m=\{2,4,6\}$ and $m=\{3,5,7\}$.

Firstly, the results obtained using the spiral model state are reported. While classically we have a second-order phase transition from Néel order (for $\kappa<\kappa_{\mathrm{cl}}$ ) to helical order (for $\kappa>\kappa_{\mathrm{cl}}$ ), where $\kappa \equiv J_{2}^{\prime} / J_{1}$, at a value $\kappa_{\mathrm{cl}}=0.5$, using the CCM we find strong indications of a shift of this critical point to values $\kappa_{c_{1}} \approx 0.80$ in the spin- $1 / 2$ quantum case and $\kappa_{c_{1}} \approx 0.62$ in the spin- 1 quantum case, as shown in Figs. 2 and 3. Thus, for example, curves such as those shown in Fig. 2 show that the Néel model state $(\phi=0)$ gives the minimum gs energy for all values of $\kappa<\kappa_{c_{1}}$, where $\kappa_{c_{1}}$ depends on the level of SUB $m-m$ approximation used, as we also observe in Fig. 3. By contrast, for values of $\kappa>\kappa_{c_{1}}$ the minimum in the energy is found to occur at a value $\phi \neq 0$. If we consider the pitch angle $\phi$ itself as an order parameter (i.e., $\phi=0$ for Néel order and $\phi \neq 0$ for spiral order) a typical scenario for a phase transition would be the appearance of a two-minimum structure for the gs energy for values of $\kappa>\kappa_{c_{1}}$, exactly as observed in Fig. 2 for both the spin- $1 / 2$ and spin-1 models in the SUB4-4 approximation. Very similar curves occur for other SUB $m-m$ approximations.

We note from Fig. 2 that for certain values of $J_{2}^{\prime}$ (or, equivalently, $\kappa$ ) CCM solutions at a given SUB $m-m$ level of approximation (viz., SUB4-4 in Fig. 2) exist only for certain ranges of spiral angle $\phi$. For example, for the pure $s=1 / 2$ squarelattice HAF $(\kappa=0)$ the CCM LSUB4 solution based on a spiral model state only exists for $0 \leq \phi \lesssim 0.20 \pi$. In this case, where the Néel solution is the stable ground state, if we attempt to move too far away from Néel collinearity the CCM equations themselves become "unstable" and simply do not have a real solution. Similarly, we see from Fig. 2(a) again that for $\kappa=1.5$; the CCM LSUB4 solution for the $s=1 / 2$ 


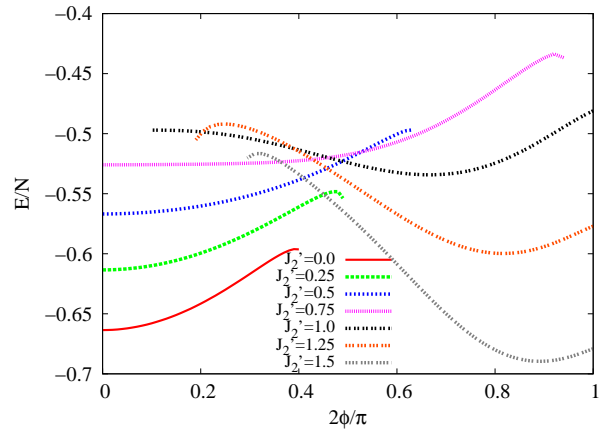

(a) $s=1 / 2$

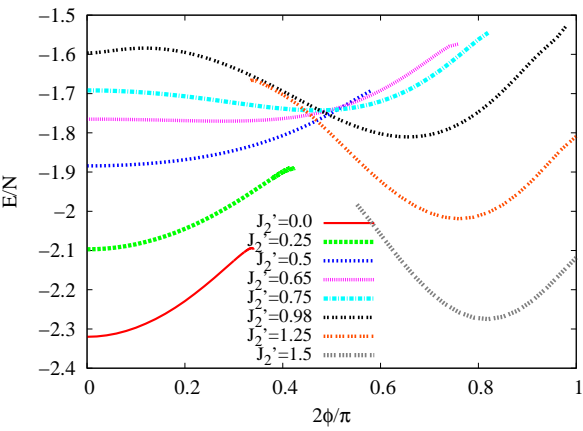

(c) $s=1$

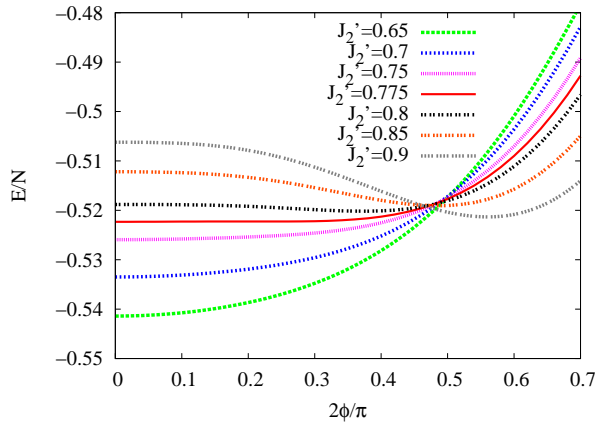

(b) $s=1 / 2$

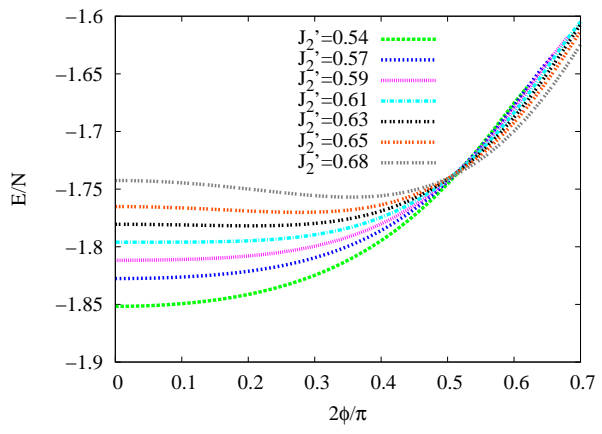

(d) $s=1$

Fig. 2. Ground-state energy per spin of the spin- $1 / 2$ and spin- $1 J_{1}-J_{2}^{\prime}$ Hamiltonians of Eq. (1) with $J_{1}=1$, using the SUB4-4 approximation of the CCM with the spiral model state, versus the spiral angle $\phi$. For the case of $s=1 / 2$, for $J_{2}^{\prime} \lesssim 0.774$ the only minimum is at $\phi=0$ (Néel order), whereas for $J_{2}^{\prime} \gtrsim 0.774$ a secondary minimum occurs at $\phi=\phi_{\mathrm{LSUB} 4} \neq 0$, which is also a global minimum, thus indicating a phase transition at $J_{2}^{\prime} \approx 0.774$ in this approximation. Similarly for the case of $s=1$, for $J_{2}^{\prime} \lesssim 0.610$ the only minimum is at $\phi=0$ (Néel order), whereas for $J_{2}^{\prime} \gtrsim 0.610$ a secondary minimum occurs at $\phi=\phi_{\mathrm{SUB} 4-4} \neq 0$, which is also a global minimum.

case exists only for $0.15 \pi \lesssim \phi \leq 0.5 \pi$. In this case the stable ground state is a spiral phase, and now if we attempt to move too close to Néel collinearity the real solution terminates.

Such terminations of CCM solutions are very common and are very well documented. ${ }^{16}$ In all such cases a termination point always arises due to the solution of the CCM equations becoming complex at this point, beyond which there exist two branches of entirely unphysical complex conjugate solutions. ${ }^{16}$ In the region where the solution reflecting the true physical solution is real there actually also exists another (unstable) real solution. However, only the (shown) upper branch 


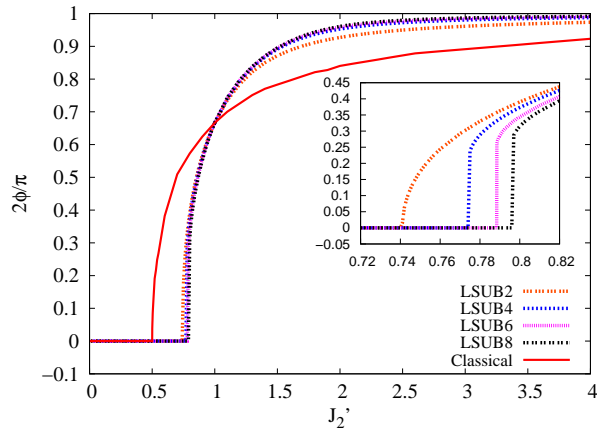

(a) $s=1 / 2$

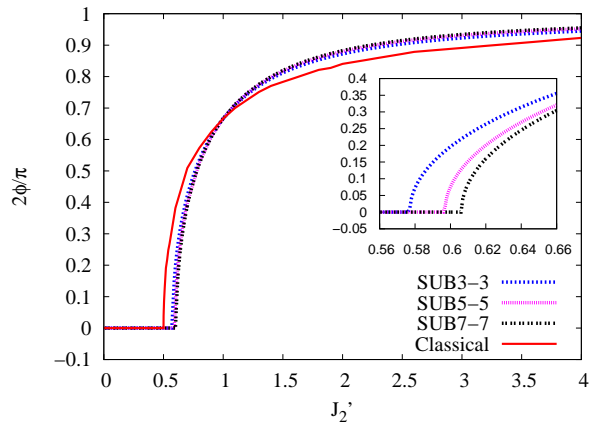

(b) $s=1$

Fig. 3. The angle $\phi=\phi_{\mathrm{SUB} m-m}$ that minimizes the energy $E_{\mathrm{SUB} m-m}(\phi)$ of the spin- $1 / 2$ and spin-1 $J_{1}-J_{2}^{\prime}$ Hamiltonians of Eq. (1) with $J_{1}=1$, in the SUB $m-m$ approximations with $m=$ $\{2,4,6,8\}$ (for $s=1 / 2$ ) and $m=\{2,4,6\}$ (for $s=1$ ), using the spiral model state, versus $J_{2}^{\prime}$. The corresponding classical result $\phi=\phi_{\mathrm{cl}}$ is shown for comparison. For $s=1 / 2$, we find in the LSUBm quantum case with $m>2$ a seemingly first-order phase transition (e.g., for LSUB8 at $J_{2}^{\prime} \approx 0.796$ where $\phi_{\text {LSUB8 }}$ jumps abruptly from zero to about $0.14 \pi$ ). By contrast, in the classical case there is a second-order phase transition at $J_{2}^{\prime}=0.5$. For $s=1$, we find in the $\mathrm{SUB} m-m$ quantum case strong evidence of a second-order phase transition (e.g., for SUB6-6 at $J_{2}^{\prime} \approx 0.612$ ), although a very weakly first-order transition cannot be entirely ruled out.

of these two solutions reflects the true (stable) physical ground state, whereas the lower branch does not. The physical branch is usually easily identified in practice as the one which becomes exact in some known (e.g., perturbative) limit. This physical branch then meets the corresponding unphysical branch at some termination point (with infinite slope in Fig. 2) beyond which no real solutions exist. The LSUBm (or SUB $m-m$ ) termination points are themselves also reflections of the quantum phase transitions in the real system, and may be used to estimate the position of the phase boundary, ${ }^{16}$ although we do not do so for this first critical point since we have more accurate criteria discussed below.

Thus, in Figs. 4 and 5 we show the CCM results for the gs energy and gs onsite magnetization, respectively, where the helical state has been used as the model state and the angle $\phi$ chosen as described above. Firstly, the gs energy (in Fig. 4(a)) for the $s=1 / 2$ case shows signs of a (weak) discontinuity in slope at the critical values $\kappa_{c_{1}}$ discussed above. Secondly, however, the gs magnetic order parameter in Fig. 5(a) for $s=1 / 2$ shows much stronger and much clearer evidence of a phase transition at the corresponding LSUB $m \kappa_{c_{1}}$ values previously observed in Fig. 3(a). The extrapolated value of $M$ shows clearly its steep drop towards a value very close to zero at $\kappa_{c_{1}}=0.80 \pm 0.01$, which is hence our best estimate of the phase transition point. From the Néel side $\left(\kappa<\kappa_{c_{1}}\right)$ the magnetization seems to approach continuously a value $M=0.025 \pm 0.025$, whereas from the spiral side $\left(\kappa>\kappa_{c_{1}}\right)$ there 


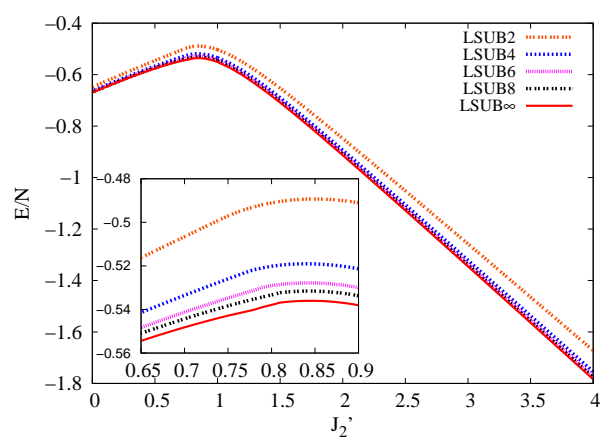

(a) $s=1 / 2$

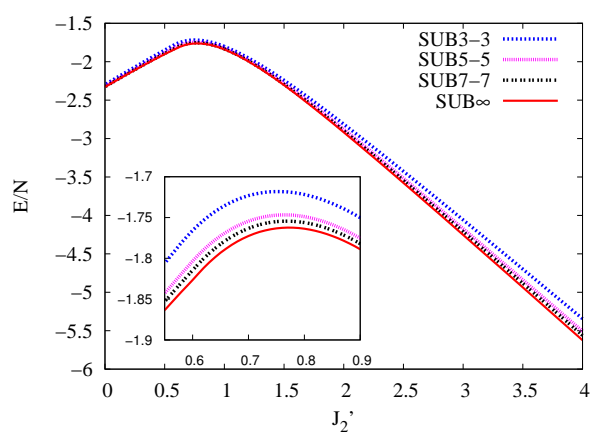

(b) $s=1$

Fig. 4. Ground-state energy per spin versus $J_{2}^{\prime}$ for the Néel and spiral phases of the spin-1/2 and spin-1 $J_{1}-J_{2}^{\prime}$ Hamiltonians of Eq. (1) with $J_{1}=1$. The CCM results using the spiral model state are shown for various SUB $m-m$ approximations $(m=\{2,4,6,8\}$ for $s=1 / 2$ and $m=\{2,4,6\}$ for $s=1)$ with the spiral angle $\phi=\phi_{\mathrm{SUB} m-m}$ that minimizes $E_{\mathrm{SUB} m-m}(\phi)$.

appears to be a discontinuous jump in the magnetization as $\kappa \rightarrow \kappa_{c_{1}}$. The transition at $\kappa=\kappa_{c_{1}}$ thus appears to be (very) weakly first-order but we cannot exclude it being second-order, since we cannot rule out the possibility of a continuous but very steep drop to zero of the on-site magnetization as $\kappa \rightarrow \kappa_{c_{1}}$ from the spiral side of the transition, for the same reasons as enunciated above in connection with our discussion of Fig. 2 and 3. These results may be compared with those for the same model of Weihong et al. ${ }^{9}$ who used a linked-cluster series expansion technique.

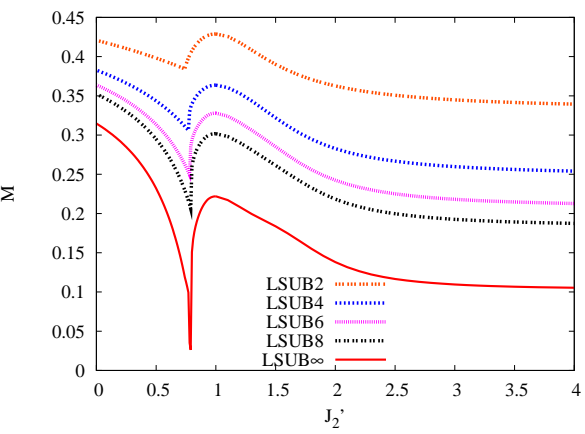

(a) $s=1 / 2$

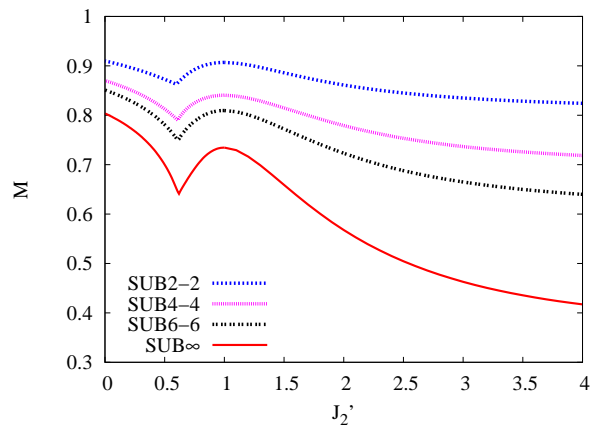

(b) $s=1$

Fig. 5. Ground-state magnetic order parameter (i.e., the on-site magnetization) versus $J_{2}^{\prime}$ for the Néel and spiral phases of the spin-1/2 and spin-1 $J_{1}-J_{2}^{\prime}$ Hamiltonians of Eq. (1) with $J_{1}=1$. The CCM results using the spiral model state are shown for various SUB $m-m$ approximations ( $m=\{2,4,6,8\}$ for $s=1 / 2$ and $m=\{2,4,6\}$ for $s=1)$ with the spiral angle $\phi=\phi_{\mathrm{SUB} m-m}$ that minimizes $E_{\mathrm{SUB} m-m}(\phi)$. 
Table 2. Ground-state energy per spin, $E / N$, and magnetic order parameter, $M$ (i.e., the on-site magnetization) for the spin- $1 / 2 \mathrm{HAF}$ on the square and triangular lattices. We show CCM results obtained for the $J_{1}-J_{2}^{\prime}$ model with $J_{1}>0$, using the spiral model state in various LSUB $m$ approximations defined on the triangular lattice geometry, for the two cases $\kappa \equiv J_{2}^{\prime} / J_{1}=0$ (square lattice HAF, $\phi=0)$ and $\kappa=1$ (triangular lattice $\mathrm{HAF}, \phi=\frac{\pi}{3}$ ).

\begin{tabular}{|c|c|c|c|c|}
\hline \multirow[t]{2}{*}{ Method } & $E / N$ & $M$ & $E / N$ & $M$ \\
\hline & \multicolumn{2}{|c|}{ square $(\kappa=0)$} & \multicolumn{2}{|c|}{ triangular $(\kappa=1)$} \\
\hline $\begin{array}{l}\text { LSUB2 } \\
\end{array}$ & -0.64833 & 0.4207 & -0.50290 & 0.4289 \\
\hline LSUB3 & -0.64931 & 0.4182 & -0.51911 & 0.4023 \\
\hline LSUB4 & -0.66356 & 0.3827 & -0.53427 & 0.3637 \\
\hline LSUB5 & -0.66345 & 0.3827 & -0.53869 & 0.3479 \\
\hline LSUB6 & -0.66695 & 0.3638 & -0.54290 & 0.3280 \\
\hline LSUB7 & -0.66696 & 0.3635 & -0.54502 & 0.3152 \\
\hline LSUB8 & -0.66816 & 0.3524 & -0.54679 & 0.3018 \\
\hline \multicolumn{5}{|c|}{ Extrapolations } \\
\hline$\overline{\operatorname{LSUB}} \infty^{a}$ & -0.66978 & 0.3148 & -0.55113 & 0.2219 \\
\hline $\operatorname{LSUB} \infty^{b}$ & -0.66974 & 0.3099 & -0.55244 & 0.1893 \\
\hline $\operatorname{LSUB} \infty^{c}$ & -0.67045 & 0.3048 & -0.55205 & 0.2085 \\
\hline $\mathrm{QMC}^{d, e}$ & $-0.669437(5)$ & $0.3070(3)$ & $-0.5458(1)$ & $0.205(10)$ \\
\hline $\mathrm{SE} f, g$ & $-0.6693(1)$ & $0.307(1)$ & $-0.5502(4)$ & $0.19(2)$ \\
\hline
\end{tabular}

\footnotetext{
${ }^{a}$ Based on LSUB $m$ results with $m=\{2,4,6,8\}$

${ }^{b}$ Based on LSUB $m$ results with $m=\{4,6,8\}$

${ }^{c}$ Based on LSUB $m$ results with $m=\{3,5,7\}$

${ }^{d}$ QMC (Quantum Monte Carlo) for square lattice (Ref. [26])

e QMC for triangular lattice (Ref. [27])

$f$ SE (Series Expansion) for square lattice (Ref. [28])

${ }^{g}$ SE for triangular lattice (Ref. [29])
}

They found that while a nonzero value of the Néel staggered magnetization exists for $0 \leq \kappa \lesssim 0.7$, the region $0.7 \lesssim \kappa \lesssim 0.9$ has zero on-site magnetization, and for $\kappa \gtrsim 0.9$ they found evidence of spiral order. Nevertheless, their results came with relatively large errors, especially for the spiral phase, and we believe that our own results are probably intrinsically more accurate than theirs.

By contrast, the gs energy shown in Fig. 4(b) for the $s=1$ case appears to be quite continuous in slope at the critical values $\kappa_{c_{1}}$ for each SUB $m-m$ level of approximation, thereby indicating a second-order transition between the Néel and helical phases. Similarly, the gs magnetic order parameter $M$ shown in Fig. 5(b) also strongly indicates a second-order transition. The extrapolated value of $M$ provides our best estimate of $\kappa_{c_{1}}=0.62 \pm 0.01$ for the $s=1 J_{1}-J_{2}^{\prime}$ model.

As a further indication of the accuracy of our results we show in Tables 2 and 3 data for the two cases of the spin- $1 / 2$ and spin- 1 HAFs on the square lattice $(\kappa=0)$ and on the triangular lattice $(\kappa=1)$. For the $s=1 / 2$ case we present our CCM results in Table 2 in various LSUB $m$ appoximations (with $2 \leq m \leq 8$ ) based on the triangular lattice geometry, and using the spiral model state, with $\phi=0$ for the square lattice and $\phi=\frac{\pi}{3}$ for the triangular lattice. Results are 
Table 3. Ground-state energy per spin, $E / N$, and magnetic order parameter, $M$ (i.e., the on-site magnetization) for the spin-1 HAF on the square and triangular lattices. We show CCM results obtained for the $J_{1}-J_{2}^{\prime}$ model with $J_{1}>0$, using the spiral model state in various $\mathrm{SUB} m-m$ approximations defined on the triangular lattice geometry, for the two cases $\kappa \equiv J_{2}^{\prime} / J_{1}=0$ (square lattice HAF, $\phi=0$ ) and $\kappa=1$ (triangular lattice HAF, $\left.\phi=\frac{\pi}{3}\right)$.

\begin{tabular}{|c|c|c|c|c|}
\hline \multirow[t]{2}{*}{ Method } & $E / N$ & $M$ & $E / N$ & $M$ \\
\hline & \multicolumn{2}{|c|}{ square $(\kappa=0)$} & \multicolumn{2}{|c|}{ triangular $(\kappa=1)$} \\
\hline SUB2-2 & -2.29504 & 0.9100 & -1.77400 & 0.9069 \\
\hline SUB3-3 & -2.29763 & 0.9059 & -1.80101 & 0.8791 \\
\hline SUB4-4 & -2.31998 & 0.8702 & -1.82231 & 0.8405 \\
\hline SUB5-5 & -2.32049 & 0.8682 & -1.82623 & 0.8294 \\
\hline SUB6-6 & -2.32507 & 0.8510 & -1.83135 & 0.8096 \\
\hline SUB7-7 & -2.32535 & 0.8492 & -1.83288 & 0.8006 \\
\hline \multicolumn{5}{|c|}{ Extrapolations } \\
\hline$\overline{\mathrm{SUB}} \infty^{a}$ & -2.32924 & 0.8038 & -1.83860 & 0.7345 \\
\hline $\mathrm{SUB} \infty^{b}$ & -2.32975 & 0.7938 & -1.83968 & 0.7086 \\
\hline $\mathrm{CCM}^{c}$ & -2.3291 & 0.8067 & & \\
\hline $\mathrm{SWT}-3^{d}$ & -2.3282 & 0.8043 & & \\
\hline $\mathrm{SE}^{e}$ & $-2.3279(2)$ & $0.8039(4)$ & & \\
\hline
\end{tabular}

$a$ Based on SUB $m-m$ results with $m=\{2,4,6\}$

$b$ Based on SUB $m-m$ results with $m=\{3,5,7\}$

${ }^{c} \mathrm{CCM}(\mathrm{SUB} \infty$ extrapolation for square lattice based on SUB $m-m$ results with $m=\{2,4,6\}$ ) (Ref. [32])

${ }^{d}$ SWT-3 (Third-order Spin-Wave Theory) for square lattice (Ref. [31])

e SE (Series Explansion) for square lattice (Ref. [28])

given for the gs energy per spin $E / N$, and the magnetic order parameter $M$. We also display our extrapolated $(m \rightarrow \infty)$ results using the schemes of Eqs. (2) and (3) with the three data sets $m=\{2,4,6,8\}, m=\{4,6,8\}$ and $m=\{3,5,7\}$. The results are seen to be very robust and consistent. For comparison we also show the results obtained for the two lattices using quantum Monte Carlo (QMC) methods ${ }^{26,27}$ and linked-cluster series expansions (SE). ${ }^{28,29}$ For the square lattice there is no dynamic frustration and the Marshall-Peierls sign rule ${ }^{30}$ applies, so that the QMC "minus-sign problem" may be circumvented. In this case the QMC results $^{26}$ are extremely accurate, and indeed represent the best available for the spin$1 / 2$ square-lattice HAF. Our own extrapolated results are in complete agreement with these QMC benchmark results, as found previously (see, e.g., Ref. [24] and references cited therein), even though the LSUB $m$ configurations are defined here on the triangular lattice geometry. Thus, we note that whereas the individual LSUBm results for the spin-1/2 square-lattice HAF do not coincide with previous results for this model (see, e.g., Ref. [24]) because previous results have been based on defining the fundamental LSUB $m$ configurations on a square-lattice geometry rather than on the triangular-lattice geometry used here, the corresponding LSUB $\infty$ extrapolations 


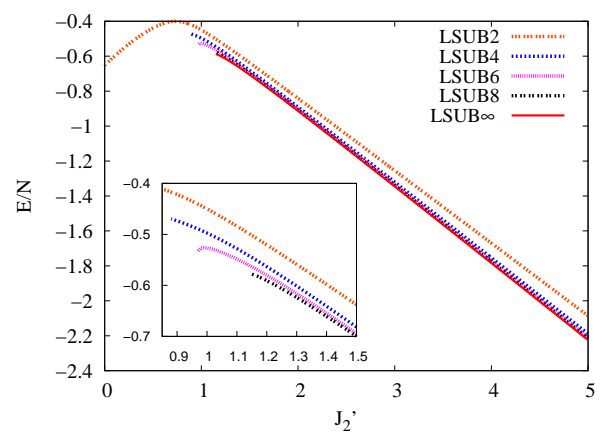

(a) $s=1 / 2$

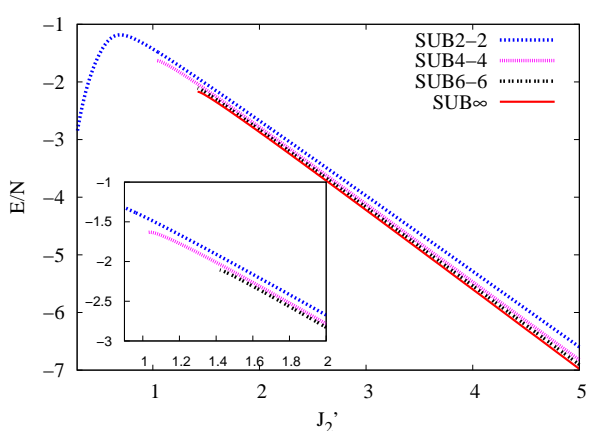

(b) $s=1$

Fig. 6. Ground-state energy per spin versus $J_{2}^{\prime}$ for the stripe-ordered phase of the spin- $1 / 2$ and spin-1 $J_{1}-J_{2}^{\prime}$ Hamiltonians of Eq. (1) with $J_{1}=1$. The CCM results using the stripe model state are shown for various SUB $m-m$ approximations $(m=\{2,4,6,8\}$ for $s=1 / 2$ and $m=\{2,4,6\}$ for $s=1)$.

in the two geometries are in complete agreement with each other.

For the $s=1$ case, our CCM results are presented in various SUB $m-m$ approximations (with $2 \leq m \leq 7)$ in Table 3 . The extrapolated results $(m \rightarrow \infty)$ using Eqs. (2) and (3) with $m=\{2,4,6\}$ and $m=\{3,5,7\}$ are also presented. For comparison we also show the results obtained for the square lattice (i.e., $\kappa=0$ ) using third-order spin-wave theory $(\mathrm{SWT}-3),{ }^{31}$ a linked-cluster series expansion $(\mathrm{SE})^{28}$ and previous CCM extrapolated $(m \rightarrow \infty)$ results based on SUB $m-m$ calculations on the square lattice. ${ }^{32}$

We turn finally to our CCM results based on the stripe state as CCM gs model state $|\Phi\rangle$. The LSUB $m$ and SUB $m-m$ configurations are again defined with respect to the triangular lattice geometry, exactly as before. Results for the gs energy and magnetic order parameter based on the collinear stripe phase are shown in Figs. 6 and 7. We see from Fig. 6(a) that some of the LSUB $n$ solutions based on the stripe state for the $s=1 / 2$ case show a clear termination point $\kappa_{t}$ of the sort discussed previously, such that for $\kappa<\kappa_{t}$ no real solution for the stripe phase exists. In particular the LSUB6 and LSUB8 solutions terminate at the values shown in Table 4. As is often the case the LSUB2 solution does not terminate, while the LSUB4 solution shows a marked change in character around the value $\kappa \approx 0.880$ that is not exactly a termination point (but, probably, rather reflects a crossing with another unphysical solution). In any event, the LSUB4 data are not shown below this value in Figs. 6(a) and 7(a). Similar termination points are seen for the $s=1$ case in Figs. 6(b) and 7(b).

The large $\kappa$ limit of the energy per spin results of Fig. 6(a) again agrees well with the exact $s=1 / 21 \mathrm{D}$ chain result of $E / N=-0.4431 J_{2}^{\prime}$ from the Bethe ansatz solution, ${ }^{12}$ just as in Fig. 4(a) for the spiral phase. However, the most important 
Table 4. The parameters $\kappa_{e}$ (the crossing point of the energy curves for the stripe and spiral phases) and $\kappa_{t}$ (the termination point of the stripe state solution) in various LSUBm approximations defined on the triangular lattice geometry, for the spin- $1 / 2$ $J_{1}-J_{2}^{\prime}$ model, with $\kappa \equiv J_{2}^{\prime} / J_{1}, J_{1}>0$. The "LSUB $\infty$ " extrapolations are explained in the text.

\begin{tabular}{ccc}
\hline Method & \multicolumn{2}{c}{$J_{2}^{\prime}$} \\
\cline { 2 - 3 } & $\kappa_{e}$ & $\kappa_{t}$ \\
\hline LSUB2 & $\infty$ & - \\
LSUB4 & 4.555 & $(0.880)$ \\
LSUB6 & 3.593 & 0.970 \\
LSUB8 & 3.125 & 1.150 \\
"LSUB $\infty$ " & $1.69 \pm 0.03$ & 1.69 \\
\hline
\end{tabular}

observation is that for all LSUB $m$ approximations with $m>2$ the curves for the energy per spin of the $s=1 / 2$ stripe phase cross with the corresponding curves (i.e., for the same value of $m$ ) for the energy per spin of the $s=1 / 2$ spiral phase at a value that we denote as $\kappa_{e}$ (as can clearly be seen in Fig. 8(a), which shows the energy difference between the stripe and spiral states for various LSUB $m$ calculations. The crossing values $\kappa_{e}$ are shown in Table 4 . Thus, for $\kappa<\kappa_{e}$ the spiral phase is predicted to be the stable phase (i.e., lies lowest in energy), whereas for $\kappa>\kappa_{e}$ the stripe phase is predicted to be the stable ground state. We thus have a clear first indication of another (first-order) quantum phase transition in the spin-1/2 $J_{1}-J_{2}^{\prime}$

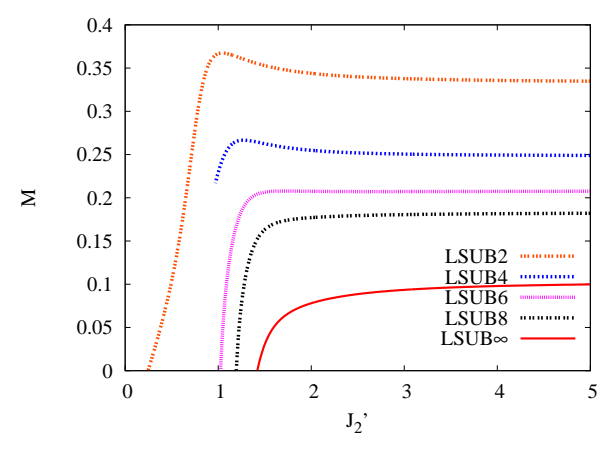

(a) $s=1 / 2$

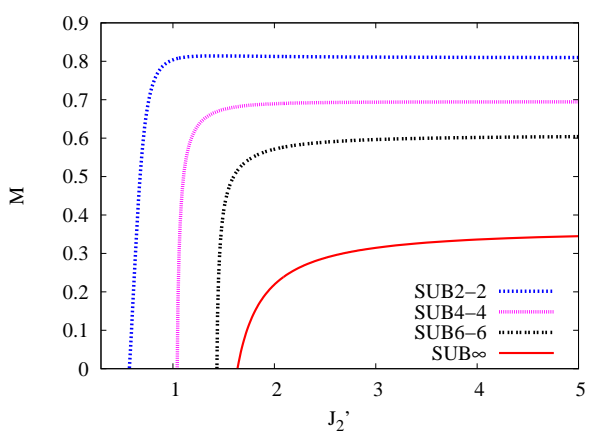

(b) $s=1$

Fig. 7. Ground-state magnetic order parameter (i.e., the on-site magnetization) versus $J_{2}^{\prime}$ for the stripe-ordered phase of the spin-1/2 and spin=1 $J_{1}-J_{2}^{\prime}$ Hamiltonians of Eq. (1) with $J_{1}=1$. The CCM results using the stripe model state are shown for various SUB $m-m$ approximations $(m=\{2,4,6,8\}$ for $s=1 / 2$ and $m=\{2,4,6\}$ for $s=1)$. 


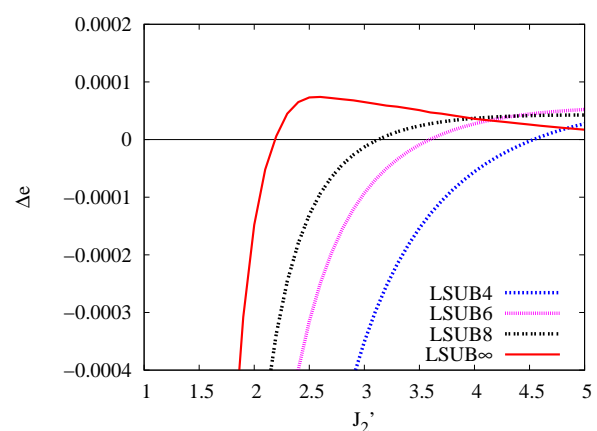

(a) $s=1 / 2$

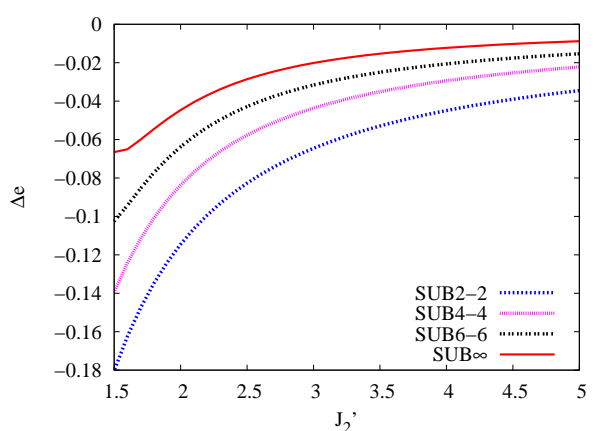

(b) $s=1$

Fig. 8. Difference between the ground-state energies per spin $(e \equiv E / N)$ of the spiral and stripe phases $\left(\Delta e \equiv e^{\text {spiral }}-e^{\text {stripe }}\right)$ versus $J_{2}^{\prime}$ for the spin-1/2 and spin-1 $J_{1}-J_{2}^{\prime}$ Hamiltonians of Eq. (1) with $J_{1}=1$. The CCM results for the energy difference using the stripe and spiral model states for various SUB $m-m$ approximations $(m=\{4,6,8\}$ for $s=1 / 2$ and $m=\{2,4,6\}$ for $s=1)$ are shown. We also show the $m \rightarrow \infty$ extrapolated results from using Eq. (2) for the two phases separately.

model at a value $\kappa=\kappa_{c_{2}}$. In summary, although it is difficult to put firm error bars on our results for our predicted second critical point, our best current estimate, based on all the above results, is $\kappa_{c_{2}}=1.8 \pm 0.4$.

For $s=1$, the large $\kappa$ limit of the energy per spin results of Fig. 6(b) agrees well with the known $1 \mathrm{D}$ chain result of $E / N=-1.4015$ obtained from a density-matrix renormalization group analysis, ${ }^{33}$ just as in Fig. $4(\mathrm{~b})$ for the spiral phase. Unlike for their spin- $1 / 2$ counterpart, the stripe phase is not a stable state for the spin-1 case because its energy lies higher than that of the spiral state for all values of $J_{2}^{\prime}$, as shown in Fig. 8(b). Hence for the $s=1$ case, there is only one quantum critical point $\kappa_{c_{1}}$ which drives the Néel phase to the helical phase.

In conclusion, we have studied the spin- $1 / 2$ and spin- $1 J_{1}-J_{2}^{\prime}$ HAF models. We have observed that as the quantum spin number $s$ is increased, the quantum critical point $\kappa_{c_{1}}$ is brought closer to the classical $(s \rightarrow \infty)$ critical point for the phase transition from the Néel phase to the helical phase. The phase transition from the Néel phase to the helical phase at $\kappa=\kappa_{c_{1}}$ for the $s=1 / 2$ case is observed to be a weakly first-order transition (although a second-order transition cannot be entirey ruled out), whereas the spin-1 case is observed to have a second-order phase transition there. There is only one quantum critical point for the $s=1$ case at $\kappa_{c_{1}}=0.62 \pm 0.01$, in contrast with the $s=1 / 2$ case where there is a second quantum critical point for the phase transition from the helical phase to the stripe phase. The two quantum critical point for the spin- $1 / 2$ case are at $\kappa_{c_{1}}=0.80 \pm 0.01$ and $\kappa_{c_{2}}=$ $1.8 \pm 0.4$. This latter result provides quantitative verification of a recent qualitative prediction of Starykh and Balents ${ }^{10}$ using a renormalization group analysis of the $J_{1}-J_{2}^{\prime}$ model that did not, however, evaluate the actual critical point. 


\section{Acknowledgement}

We thank the University of Minnesota Supercomputing Institute for Digital Simulation and Advanced Computation for the grant of supercomputing facilities, on which we relied heavily for the numerical calculations reported here.

\section{References}

1. S. Sachdev, in Low Dimensional Quantum Field Theories for Condensed Matter Physicists, ed. Y. Lu, S. Lundqvist, and G. Morandi (World Scientific, Singapore 1995).

2. J. Richter, J. Schulenburg, and A. Honecker, in Quantum Magnetism, Lecture Notes in Physics Vol. 645, ed. U. Schollwöck, J. Richter, D.J.J. Farnell, and R.F. Bishop, (Springer-Verlag, Berlin, 2004), p. 85.

3. G. Misguich and C. Lhuillier, in Frustrated Spin Systems, ed. H.T. Diep (World Scientific, Singapore, 2005), p. 229.

4. R. F. Bishop, P. H. Y. Li, R. Darradi, and J. Richter, J. Phys.: Condens. Matter 20, 255251 (2008).

5. R. F. Bishop, P. H. Y. Li, R. Darradi, J. Schulenburg, and J. Richter, Phys. Rev. B 78, 054412 (2008).

6. C. J. Gazza and H. A. Ceccatto, J. Phys.: Condens. Matter 5, L135 (1993).

7. A. E. Trumper, Phys. Rev. B 60, 2987 (1999).

8. J. Merino, R. H. McKenzie, J. B. Marston, and C. H. Chung, J. Phys.: Condens. Matter 11, 2965 (1999).

9. Zheng Weihong, R. H. McKenzie, and R. R. P. Singh, Phys. Rev. B 59, 14367 (1999).

10. O. A. Starykh and L. Balents, Phys. Rev. Lett. 98, 077205 (2007).

11. T. Pardini and R. R. P. Singh, Phys. Rev. B 77, 214433 (2008).

12. H. A. Bethe, Z. Phys. 71, 205 (1931).

13. J. Villain, J. Phys. (Paris) 38, 385 (1977); J. Villain, R. Bidaux, J. P. Carton, and R. Conte, ibid. 41, 1263 (1980); E. Shender, Sov. Phys. JETP 56, 178 (1982).

14. R. F. Bishop, Theor. Chim. Acta 80, 95 (1991).

15. R. F. Bishop, in Microscopic Quantum Many-Body Theories and Their Applications, edited by J. Navarro and A. Polls, Lecture Notes in Physics 510 (Springer-Verlag, Berlin, 1998), p.1.

16. D. J. J. Farnell and R. F. Bishop, in Quantum Magnetism, edited by U. Schollwöck, J. Richter, D. J. J. Farnell, and R. F. Bishop, Lecture Notes in Physics 645 (SpringerVerlag, Berlin, 2004), p.307.

17. D. J. J. Farnell, R. F. Bishop, and K. A. Gernoth, Phys. Rev. B 63, 220402(R) (2001).

18. S. E. Krüger, J. Richter, J. Schulenburg, D. J. J. Farnell, and R. F. Bishop, Phys. Rev. B 61, 14607 (2000).

19. D. Schmalfuß, R. Darradi, J. Richter, J. Schulenburg, and D. Ihle, Phys. Rev. Lett. 97, 157201 (2006).

20. C. Zeng, D. J. J. Farnell, and R. F. Bishop, J. Stat. Phys. 90, 327 (1998).

21. R. Darradi, J. Richter, and D. J. J. Farnell, Phys. Rev. B 72, 104425 (2005).

22. D. J. J. Farnell, R. F. Bishop, and K. A. Gernoth, J. Stat. Phys. 108, 401 (2002).

23. We use the program package "Crystallographic Coupled Cluster Method" (CCCM) of D. J. J. Farnell and J. Schulenburg, see http://www-e.unimagdeburg.de/jschulen/ccm/index.html.

24. D. J. J. Farnell and R. F. Bishop, Int. J. Mod. Phys. B 22, 3369 (2008).

25. P. M. Morse and H. Feshbach, Methods of Theoretical Physics, Part II (McGraw-Hill, New York, 1953). 
16 R. F. Bishop et al

26. A. W. Sandvik, Phys. Rev. B 56, 11678 (1997).

27. L. Capriotti, A. E. Trumper, and S. Sorella, Phys. Rev. Lett. 82, 3899 (1999).

28. Zheng Weihong, J. Oitmaa, and C. J. Hamer, Phy. Rev. B 43, 8321 (1991).

29. W. Zheng, J. O. Fjaerestad, R. R. P. Singh, R. H. McKenzie, and R. Coldea, Phys. Rev. B 74, 224420 (2006).

30. W. Marshall, Proc. R. Soc. London, Ser. A 232, 48 (1955).

31. C. J. Hamer, W. H. Zheng, and P. Arndt, Phys. Rev. B 46, 6276 (1992).

32. D. J. J. Farnell, K. A. Gernoth, and R. F. Bishop, Phys. Rev. B 64, 172409 (2001).

33. S. R. White and D. A. Huse, Phys. Rev. B 48, 3844 (1993). 